ESTUDIO DE CASO

\title{
DETERMINACIÓN DEL COSTO DE LA CRIANZA DE TERNERAS DESDE EL NACIMIENTO HASTA EL DESTETE EN UNA LECHERÍA COMERCIAL ESPECIALIZADA ${ }^{1}$
}

\author{
Jorge Alberto Elizondo-Salazar ${ }^{2}$, Alexandra María Vargas-Ramírez $^{3}$
}

\section{RESUMEN}

El objetivo del presente trabajo fue determinar el costo económico asociado a la cría de reemplazos desde el nacimiento hasta el destete en una finca lechera comercial. Las determinaciones se realizaron en una finca de lechería especializada en San Ramón de Tres Ríos, Cartago. Durante el periodo del estudio se utilizaron datos de 23 terneras de la raza Holstein de cero a cuatro meses de edad. Las terneras fueron alimentadas según el método ya establecido en la finca. Para determinar el costo económico asociado a la cría de reemplazos desde el nacimiento hasta los cuatro meses de edad bajo el sistema de crianza utilizado, se llevó un registro diario por animal de cada gasto realizado, incluyendo alimentación, productos veterinarios y mano de obra, entre otros. Se determinó que el costo total promedio para criar una ternera hasta los cuatro meses de edad bajo el sistema de crianza utilizado en la finca es de $\mathbb{2} 221.287,88(\$ 442,97)$, el cual puede aumentar en un rango entre los $\$ 467,34$ y $\not 18.692,30$ (\$0,94 y $\$ 37,42$, respectivamente).

El rubro de mayor impacto del total del costo promedio de producción resultó ser la alimentación con un $64,45 \%$, del cual un $24,57 \%$ corresponde al gasto de alimento balanceado promedio, seguidamente se encuentra el costo promedio por concepto de lactoremplazador y de leche íntegra los cuales representan un 20,53 y 13,76\%, respectivamente. La mano de obra requerida para el cuido de estos animales resultó ser el segundo costo de mayor importancia con una participación del 18,89\% siendo específicamente la mano de obra del ternerero la de mayor impacto con un $18,33 \%$.

Palabras clave: valor, terneras, ganado lechero, reemplazos

\footnotetext{
${ }^{1}$ Inscrito en la Vicerrectoría de Investigación de la Universidad de Costa Rica. Proyecto 737-B4-222.

${ }^{2}$ Universidad de Costa Rica. Facultad de Ciencias Agroalimentarias. Estación Experimental Alfredo Volio Mata. Autor para correspondencia: jorge.elizondosalazar@ucr.ac.cr

${ }^{3}$ Universidad de Costa Rica. Graduada licenciada de la Escuela de Zootecnia.

Recibido: 6 de abril 2014

Aceptado: 3 de julio 2015
} 


\section{ABSTRACT}

Determination of calf rearing costs from birth to weaning in a commercial specialized dairy farm. The aim of this study was to determine the economic cost associated with rearing of replacements from birth to weaning on a commercial dairy farm. The data were collected from a specialized dairy farm in San Ramon de Tres Rios, Cartago. During the study period data from 23 Holstein heifer calves from zero to four months of age were used. Calves were fed according to the method already established on the farm. To determine the economic cost associated with rearing from birth to four months of age, under the farm system, an expense record was kept for each animal, the record included feed, veterinary products, and labor cost among others. It was determined that the average total cost to raise a calf from birth to four months of age was $\$ 221,287.88$ (\$442.97), which can increase in a range between $\not 467.34$ and $\not 18,692.30$ (\$ 0.94 and \$ 37.42 , respectively). Feed cost impacted the most the total cost $(64.45 \%)$, of which $24.57 \%$ corresponded to concentrate, followed by milk replacer and whole milk, which accounted for 20.53 and $13.76 \%$, respectively. Labor required for the care of these animals proved to be the second most important cost with a share of $18.89 \%$ of which $18.33 \%$ corresponded to the person taking care of the calves.

Keywords: Value, calves, dairy cattle, replacements

\section{INTRODUCCIÓN}

A lo largo de muchos años de historia, la vaca lechera ha hecho grandes contribuciones a la alimentación y salud del ser humano. Económicamente, la industria lechera juega un papel muy importante a nivel mundial y nuestro país es una muestra muy clara de esta situación. Así por ejemplo, en Costa Rica existen alrededor de 45.782 fincas, de las cuales el $59 \%$ se dedican a la producción láctea, lo que crea alrededor de 46.147 empleos directos y generando el $74,8 \%$ del volumen de las exportaciones del sector pecuario (Cámara Nacional de Productores de Leche, 2014). 
Nuestro país pasó de producir 308 millones de kilogramos de leche en el año 1980 a 1055 en el 2013 (Cámara Nacional de Productores de Leche, 2014), lo que representa un incremento del $342 \%$. Este aumento en la producción, junto con mejoras en tecnologías y técnicas de manejo, ha puesto una presión en la crianza y desarrollo de reemplazos, especialmente cuando se considera que es uno de los aspectos esenciales para el futuro comportamiento de la finca y la rentabilidad de la misma.

Debido a la naturaleza del manejo de los animales de reemplazo, una explotación lechera debe invertir en alimentación, mano de obra, medicamentos y capital financiero, entre otros gastos, por aproximadamente dos años, sin recibir ningún retorno económico, por lo que determinar el costo asociado a criar estos animales debe ser una parte esencial de cualquier sistema de explotación lechera.

Heinrichs (1993) reportó que en los Estados Unidos, el desarrollo de reemplazos representa aproximadamente el $20 \%$ del total de gastos de la operación de una finca lechera y es el segundo costo más alto después de la alimentación. Por lo tanto, minimizar la inversión en esta etapa de vida, mientras se mantiene la integridad productiva, debe ser un objetivo primordial en el manejo de animales de reemplazo.

Las operaciones lecheras cuentan con una serie de recursos y objetivos específicos, por lo que la manera más económica de obtener reemplazos está determinada solamente por el análisis individual de costos de cada explotación. En Costa Rica, existen muy pocos estudios que hayan determinado este tipo de información. Rivera (2000) al analizar el retorno de la inversión en la crianza de novillas Jersey a primer parto en ocho fincas de la zona de altura de la Meseta Central, estableció que existe una gran variación en el costo de producción de reemplazos e indicó que el valor de crianza y desarrollo de estos animales varió de $\$ 212.354,0(\$ 679,03)$ a $\$ 426.254,7(\$ 1.363,01)$ por animal. El promedio para la zona fue de $\not 302.094,7(\$ 965,99)$ y donde el costo diario promedio de la crianza de reemplazos en la zona fue de $\mathbb{4} 423,73(\$ 1,35)$.

El estudio realizado por Rivera (2000), expone que el mayor costo de crianza en terneras es la alimentación, el cual representó entre un 31,8 a un $64,3 \%$ de los costos totales. El autor explica que esto se debe a que en esta etapa se utilizan alimentos balanceados de mayor calidad nutricional, los cuales en la mayoría de los casos son productos de importación como los pre-iniciadores e iniciadores, además se utilizan otros productos de 
alto costo como la leche íntegra, lactoreemplazadores, suplementos vitamínicos, minerales y forraje.

El objetivo del presente trabajo fue determinar el costo económico asociado a la cría de reemplazos desde el nacimiento hasta el destete en una finca lechera comercial.

\section{MATERIALES Y MÉTODOS}

\section{Ubicación de la finca}

El presente trabajo se realizó en la sección de crianza de terneras de una finca lechera comercial especializada ubicada en San Ramón de Tres Ríos, provincia de Cartago, Costa Rica. La explotación se ubica a una altura promedio de $1.650 \mathrm{msnm}$, con una precipitación promedio anual de $2.000 \mathrm{~mm}$ y temperatura anual $14-15^{\circ} \mathrm{C}$ en la mañana y $25^{\circ} \mathrm{C}$ durante la tarde.

\section{Población animal utilizada}

Durante el periodo del estudio se utilizaron datos de 23 terneras de la raza Holstein de cero a cuatro meses de edad. Los animales fueron separados de sus madres desde el nacimiento y alojados en cunas individuales de metal de 2,0 x 1,2 m, con piso de cemento y cama de burucha.

\section{Plan de alimentación}

Las terneras fueron alimentadas según el método ya establecido en la finca, en donde los primeros dos días de vida se les suministraron $4 \mathrm{~L}$ de calostro, $(2 \mathrm{~L}$ a.m. y $2 \mathrm{~L}$ p.m.). De los 3 a 30 días de edad, se les ofreció $4 \mathrm{~L}$ de leche íntegra suministrada en balde a $38^{\circ} \mathrm{C}$. A partir de los 30 días de edad se les ofreció lactoreemplazador (97,00\% MS; 20,50\% PC y $16,00 \%$ grasa) en balde dos veces al día ( 2 L a.m. y 2 L p.m.) reconstituido a razón de $0,125 \mathrm{~kg}$ en $0,9 \mathrm{~L}$ de agua a $40^{\circ} \mathrm{C}$. A partir del tercer día de nacidas se les ofreció también alimento balanceado tipo pre-inicio (88,00\% MS; 22,00\% PC; 3,00\% EE y 1,55 Mcal ENg) hasta que alcanzaron un consumo de $0,5 \mathrm{~kg}$ de materia fresca (MF) por día. 
Una vez que alcanzaron dicho consumo, se les suministró alimento balanceado tipo inicio (88,00\% MS; 17,00\% PC; 3,00\% EE y 1,93 Mcal ENg), hasta alcanzar un consumo de 1,5 $\mathrm{kg}$ de MF. Finalmente alcanzado el consumo anterior, se les suministró alimento balanceado tipo desarrollado (87,00\% MS; 16,00\% PC; 3,00\% EE y 1,65 Mcal ENg) hasta que lograron un consumo de 2,0 kg MF por día, cantidad que se mantuvo fija durante el resto de la etapa de crianza.

Las terneras fueron destetadas entre las 11 y 15 semanas de vida, cuando alcanzaron un consumo de $1,5 \mathrm{~kg}$ MF de alimento balanceado tipo inicio. A partir de los 30 días de vida aproximadamente se les suministró heno de pasto Transvala (Digitaria decumbens) (89,00\% MS; 4,72\% PC; 1,70\% EE y 1,17 Mcal ENg) a libre consumo.

Para establecer adecuadamente el costo de la alimentación y del agua, se llevó un registro diario de la cantidad ofrecida y del rechazo por cada animal, para el alimento balanceado, el forraje y el agua.

\section{Estimación de costos desde el nacimiento hasta los cuatro meses de edad}

Para determinar el costo económico asociado a la cría de reemplazos desde el nacimiento hasta los cuatro meses de edad bajo el sistema de crianza de la finca, se llevó un registro diario por animal de cada gasto realizado referente a la crianza. Se contabilizaron: litros de lactoreemplazador, kilogramos de alimento balanceado, kilogramos de forraje, suplementos nutricionales, vacunas, antibióticos, desparasitaciones, tratamientos, sueros, burucha, productos de limpieza y desinfección, agua, horas de trabajo del encargado de la crianza (ternerero), arete y costo de registro. Todos los costos asociados a las compras, se tomaron de las facturas emitidas por el vendedor o negocio donde el productor realizó las compras. El costo de la mano de obra se consideró con el salario reportado a la Caja Costarricense del Seguro Social. Para el establecimiento de los costos en este trabajo, no se consideró la depreciación ni mantenimiento de las instalaciones, ya que estaban depreciadas por haber cumplido la vida útil y al ser instalaciones antiguas, no se le daba mantenimiento.

Una vez obtenido el costo de producción promedio de una ternera, se procedió a contrastarlo con los costos de producción promedio derivados de sí hipotéticamente la 
ternera fuera destetada a los tres o dos meses de edad. Los costos de producción de una ternera destetada a los tres o dos meses de edad se obtuvieron mediante la reducción de los costos por concepto de lactoreemplazador y mano de obra del operario encargado de las terneras, manteniendo invariables los costos restantes y no se consideraron otros costos, ya que no existían en esta explotación.

Para estandarizar los costos de producción estimados para la crianza de terneras, se utilizó el valor promedio de \$499,55 obtenido entre la compra y la venta del dólar estadounidense según el tipo de cambio anunciado por el Banco Central de Costa Rica (2015) para el 23 de setiembre del 2013. Además, con el fin de poder comparar los costos obtenidos en el presente estudio expresados en dólares con aquellos costos reportados por Rivera (2000) para la crianza de reemplazos desde el nacimiento hasta el destete expresados en colones, se procedió a transformar estos últimos a dólares, tomando como referencia el valor promedio de $\$ 312,73$ obtenido entre la compra y la venta del dólar estadounidense establecido para el día 23 de setiembre del año 2000 (Banco Central de Costa Rica, 2015).

\section{RESULTADOS Y DISCUSIÓN}

\section{Costos de producción}

Los resultados de los costos de producción promedio de las terneras durante los primeros cuatro meses de vida se muestran en el Cuadro 1. Se determinó que el costo total promedio para criar una ternera hasta los cuatro meses de edad bajo el sistema de crianza de la finca fue de $\not 221.287,88(\$ 442,97)$, el cual puede aumentar en un rango entre los $\$ 467,34$ y $\not 18.692,30$ (\$0,94 y $\$ 37,42$, respectivamente). Este intervalo corresponde a aquel costo extra para las terneras que fueron sometidas a algún tratamiento o cuidado específico debido a un estado de salud en particular como tratamiento contra diarreas y afecciones respiratorias. Las terneras que presentaron problemas de diarreas fueron las que más incrementaron los costos de crianza debido a la aplicación de medicamentos anti-timpánicos, antibióticos y terapias de electrolitos con componentes nutritivos y antimicrobianos, en comparación con las que sólo fueron tratadas con antibióticos y antihistamínicos debido a afecciones respiratorias. 
Cuadro 1. Costos de producción promedio de una ternera de cero a cuatro meses de edad en una finca lechera comercial. San Ramón de Tres Ríos, Cartago.

\begin{tabular}{|c|c|c|c|}
\hline Rubro & Colones & Dólares & Porcentaje \\
\hline \multicolumn{4}{|l|}{ Alimentación } \\
\hline Alimento balanceado & $54.379,37$ & 108,86 & 24,57 \\
\hline Forraje & $9.164,75$ & 18,35 & 4,14 \\
\hline Leche íntegra & $30.459,80$ & 60,97 & 13,76 \\
\hline Lactoreemplazador & $45.423,74$ & 90,93 & 20,53 \\
\hline Agua & 8,26 & 0,02 & 0,00 \\
\hline Suplemento calostral & $3.125,00$ & 6,26 & 1,41 \\
\hline Complejo vitamínico $A, D$ y E & 67,20 & 0,13 & 0,03 \\
\hline Total alimentación promedio & $142.628,12$ & 285,51 & 64,45 \\
\hline \multicolumn{4}{|l|}{ Manejo sanitario } \\
\hline Desinfectante antiviral & $2.350,00$ & 4,70 & 1,06 \\
\hline Cal dolomita & 93,30 & 0,19 & 0,04 \\
\hline Yodo (7\%) & 286,00 & 0,57 & 0,13 \\
\hline Antibióticos & 191,60 & 0,38 & 0,09 \\
\hline Camas & $11.900,00$ & 23,82 & 5,38 \\
\hline Desparasitante oral & 47,60 & 0,10 & 0,02 \\
\hline Vacunas & $19.085,00$ & 38,20 & 8,62 \\
\hline Total manejo sanitario promedio & $33.953,00$ & 67,97 & 15,34 \\
\hline \multicolumn{4}{|l|}{ Identificación } \\
\hline Arete & 395,00 & 0,79 & 0,18 \\
\hline Registro Asociación Criadores Holstein & $2.500,00$ & 5,00 & 1,13 \\
\hline Total identificación promedio & $2.895,00$ & 5,80 & 1,31 \\
\hline \multicolumn{4}{|l|}{ Mano de obra } \\
\hline Ternerero & $40.561,27$ & 81,20 & 18,33 \\
\hline Vacunador & $1.250,00$ & 2,50 & 0,56 \\
\hline Total mano de obra promedio & $41.811,27$ & 83,70 & 18,89 \\
\hline COSTO TOTAL PROMEDIO & $221.287,88$ & 442,97 & 100,00 \\
\hline
\end{tabular}


El costo total promedio obtenido en el presente estudio prácticamente duplica al costo total reportado en el estudio realizado por Rivera (2000). Dicho estudio se realizó en 8 fincas de lechería especializada en la Meseta Central, donde se determinó que los costos de crianza de una ternera Jersey en el año 2000 desde el nacimiento hasta el destete con una edad promedio de 3,2 meses se encontraban en un intervalo entre los $\$ 172,56$ y $\$ 275,69$, con un valor promedio de $\$ 226,99 \pm 35,86$. Dicho autor menciona que los rubros de alimentación, mano de obra y fertilización son los que más incrementan los costos de crianza y desarrollo reemplazos, y que a mayor duración de la etapa se obtendrá un mayor costo de la misma. La diferencia de costos tan importante entre los dos estudios, se debe principalmente al aumento en los costos de mano de obra y de todos los insumos.

Es importante hacer notar que el presente estudio, no consideró la depreciación del equipo ni de las edificaciones, como si lo hizo el estudio de Rivera (2000), ya que estaban depreciadas por haber cumplido la vida útil y al ser instalaciones antiguas, no se les daba mantenimiento.

El rubro que más impactó el costo total promedio de producción resultó ser la alimentación con un $64,45 \%$, del cual un $24,57 \%$ corresponde al gasto de alimento balanceado, seguidamente se encuentra el costo por concepto de lactoreemplazador y de leche íntegra los cuales representan un 20,53 y 13,76\%, respectivamente. Este tipo de alimentos balanceados tipo pre-inicio e inicio así como el lactoreemplazador utilizados para la crianza de estos animales son sumamente costosos, ya que para su elaboración se utilizan materias primas que no son producidas en Costa Rica.

La mano de obra requerida para el cuido de estos animales resultó ser el segundo costo de mayor importancia con una participación del 18,89\% siendo específicamente la mano de obra del ternerero la de mayor impacto con un $18,33 \%$. Se estimó que el ternerero dedica, por cada animal de cero a cuatro meses de edad, aproximadamente 0,3 horas al día en tareas tales como alimentación y limpieza de cunas, para un total aproximado de 35,7 horas durante este periodo. En cuanto a la mano de obra del vacunador se deriva de una única aplicación de un tratamiento durante este periodo convirtiéndolo en un costo fijo. Para Costa Rica, la Cámara Nacional de Productores de Leche (2014) en la estructura porcentual de costos para la producción de leche a nivel de finca, indica que el costo de la mano de obra es también el segundo rubro en importancia y representa el $20,8 \%$.

Nutrición Animal Tropical 9(2): 1-10. ISSN: 2215-3527/ 2015 
Los costos promedio por concepto de manejo sanitario en el presente estudio ocuparon el tercer lugar con un 15,34\%.

Acortar la edad de destete de las terneras se presenta como una alternativa para reducir los costos de crianza de los animales, ya que se disminuiría los costos por concepto de mano de obra por parte del ternerero así como la cantidad de leche y/o lactoreemplazador utilizados durante esta etapa. En el Cuadro 2 se presenta la reducción en el costo promedio de mano de obra del ternero y una disminución en el costo por concepto de lactoreemplazador, así como su efecto en el costo total promedio de crianza suponiendo que las terneras fuesen destetadas a los dos o a los tres meses de edad, asumiendo que todos los demás costos de producción se mantienen constantes en ambos casos.

Cuadro 2. Costos de producción promedio de una ternera que se desteta a los dos o a los tres meses de edad.

\begin{tabular}{ccccccc}
\hline \multirow{2}{*}{ Rubro } & \multicolumn{2}{c}{2 meses } & & \multicolumn{2}{c}{3 meses } \\
\cline { 2 - 3 } \cline { 5 - 6 } Mano de obra ternerero & $31.187,86$ & 62,43 & & $36.385,84$ & 72,84 \\
Lactoreemplazador & $22.995,61$ & 46,03 & & $40.672,57$ & 81,42 \\
COSTO TOTAL PROMEDIO & $190.557,34$ & 381,46 & & $213.432,28$ & 427,25 \\
\hline
\end{tabular}

En caso de que se redujera la edad de destete de las terneras a tres meses, se obtendría una disminución de los costos por concepto de mano de obra del ternerero de $\$ 8,36$ y una reducción de los costos por concepto de consumo de lactoreemplazador de $\$ 9,51$, lo que representaría una reducción del 3,55\% del costo total de crianza obtenido bajo el sistema actual que impera en la finca. Sin embargo, si los animales se destetaran a los dos meses de edad, se lograría una disminución del total del costo de crianza de un 13,89\%, ya que el costo por concepto de mano de obra del ternerero se reduciría en $\$ 18,77$ y el costo por concepto de consumo de lactoreemplazador disminuiría en $\$ 44,9$. 


\section{CONSIDERACIONES FINALES}

El rubro de mayor impacto del total del costo promedio de producción resultó ser la alimentación con un $64,45 \%$, del cual un $24,57 \%$ corresponde al gasto por concepto de consumo de alimento balanceado.

El segundo costo de mayor importancia fue la mano de obra requerida para la crianza de las terneras con una participación del $18,89 \%$, siendo específicamente la mano de obra del ternerero la de mayor impacto con un $18,33 \%$.

Los costos de crianza de una ternera de cero a cuatro meses de edad en esta finca en particular, se pueden reducir en un $3,55 \%$ si es destetada a los tres meses o bien pueden reducirse en un $13,89 \%$ si es destetada a los dos meses de edad.

\section{LITERATURA CITADA}

Banco Central De Costa Rica. 2015. Tipo cambio de compra y de venta del dólar de los Estados Unidos de América. Disponible en: http://indicadoreseconomicos.bccr.fi.cr/indicadoreseconomicos/cuadros/frmvercatcuadr o. $\operatorname{aspx}$ ?CodCuadro=400.

Cámara Nacional de Productores de Leche. 2014. Situación actual y perspectivas del sector lácteo nacional. XX Congreso Nacional Lechero. Realizado el 21 y 22 de Octubre del 2014. Heredia, Costa Rica.

Heinrichs, A.J. 1993. Raising dairy replacements to meet the needs of the 21 st century. J. Dairy Science. 76:3179-3187.

Rivera, A. 2000. Determinación del retorno de la inversión en la crianza y desarrollo de novillas Jersey a primer parto en la zona de altura de la Meseta Central de Costa Rica. Tesis para optar al grado de licenciatura. Escuela de Zootecnia. Universidad de Costa Rica. San Pedro de Montes de Oca. 